\title{
GROUP CLASSIFICATION OF COUPLED DIFFUSION SYSTEM WITH APPLICATIONS IN SOIL SCIENCE
}

\author{
M. Molati ${ }^{1}$ and F.M. Mahomed \\ Centre for Differential Equations, Continuum Mechanics and Applications, \\ School of Computational and Applied Mathematics, \\ University of the Witwatersrand, Johannesburg, \\ Private Bag 3, Wits 2050, South Africa \\ m.molati@gmail.com, Fazal.Mahomed@wits.ac.za
}

\begin{abstract}
We perform a complete group classification of a coupled system of diffusion equations with applications in soil science. The canonical forms of the lowdimensional Lie algebras and the Lie algebras of higher dimension provide a means to specify the diffusion coefficients completely.
\end{abstract}

Keywords- Group classification, Coupled diffusion, Lie algebra.

Dedicated to Professor David P. Mason on the occasion of his 65th birthday.

\section{INTRODUCTION}

A group classification for a general second-order system of diffusion equations based on Lie algebras of low dimension was performed in [1]. We follow exactly the procedure used in this classification for the current problem. Briefly, the classification procedure involves the utilization of the structure of the low-dimensional Lie algebras and the Lie algebras of higher dimension to find the symmetry operators admitted by the underlying equation or system. The procedure is continued until the functional forms of the unknown functions are completely specified. That is, the equivalence group is used to obtain the canonical forms of the symmetry operators which satisfy the model under consideration. Even though this procedure was suggested in $[2,3]$ for partial differential equations (PDEs), a much earlier work on ordinary differential equations (ODEs) using these ideas was done in [4]. We use the results on classification of solvable Lie algebras by Mubarakzyanov [5] reported in Basarab-Horwarth [3].

The theory of coupled diffusion appears in the works of Philip and De Vries [6], De Vries [7] in the late fifties and later by many authors, such as Jury et al [8].

\footnotetext{
${ }^{1}$ Permanent address: Department of Mathematics and Computer Science, National University of Lesotho, P O Roma 180, Lesotho.
} 
The coupled diffusion is described by the partial differential equations $[9,10,11]$, i.e.,

$$
\begin{aligned}
& u_{t}=\left[f^{1}(u, v) u_{x}+f^{2}(u, v) v_{x}\right]_{x}, \\
& v_{t}=\left[f^{3}(u, v) u_{x}+f^{4}(u, v) v_{x}\right]_{x},
\end{aligned}
$$

where $u(t, x)$ and $v(t, x)$ are the soil temperature and volumetric water content respectively. The variable $x$ measures the depth of the soil and $t$ is the time. The arbitrary functions $f^{1}, \ldots, f^{4}$ are the diffusion coefficients.

Wiltshire et al $[11,13]$ investigated the Lie symmetries of a simplified model of the coupled diffusion system (1) written in the form

$$
\mathbf{y}_{t}=\left[\Lambda(\mathbf{y}) \mathbf{y}_{x}\right]_{x}, \mathbf{y}=\left\{y_{i}\right\} \quad(i=1,2, \ldots, n),
$$

where $\Lambda(\mathbf{y})$ is a square matrix of diffusion coefficients. These investigations involve rewriting a system of coupled diffusion equations into a single equation in which the diffusion coefficients are written in a matrix form. Thus the resulting determining equations to be solved for symmetries are in terms of the systems of matrix equations. The matrix diffusion equation (2) is a generalized form of the extensively studied one-dimensional nonlinear heat conduction equation the group properties of which were first considered in [12]. Therefore the group classification procedure on Eq. (2) is analogous to the analysis performed in the cited reference and those that followed.

In this work, our goal is to derive the Lie symmetries of system (1) as it is and see whether a comparison with those of the simplified model (2) can be established. The non classical (potential) symmetries of the coupled system (1) were generated in [13]. The generation of the determining equations and the manipulation of them are with the aid of the YaLie software package [14].

\section{EQUIVALENCE GROUP AND GENERATOR OF SYMMETRY}

The equivalence group for system (1) was obtained in [10] using the infinitesimal approach. It can shown that the direct method yields the same result. However, instead of using these equivalence transformations we choose the equivalence group of the form

$$
\bar{t}=T(t), \quad \bar{x}=X(x), \quad \bar{u}=U(u, v), \quad \bar{v}=V(u, v),
$$

where the functions $T, X, U$ and $V$ satisfy the conditions

$$
T_{t} \neq 0, \quad X_{x} \neq 0, \quad \frac{D(U, V)}{D(u, v)} \neq 0
$$


Using the basic Lie technique $[15,16,17]$ we seek a symmetry generator of the form

$$
\Gamma=\xi^{1}(t, x, u, v) \partial_{t}+\xi^{2}(t, x, u, v) \partial_{x}+\eta^{1}(t, x, u, v) \partial_{u}+\eta^{2}(t, x, u, v) \partial_{v}
$$

The operator $\Gamma$ is a generator of symmetry group of system (1) if and only if

$$
\begin{gathered}
\Gamma^{[2]}\left(u_{t}-f^{1} u_{x x}-f^{2} v_{x x}-u_{x}\left[f_{u}^{1} u_{x}+f_{v}^{1} v_{x}\right]-v_{x}\left[f_{u}^{2} u_{x}+f_{v}^{2} v_{x}\right]\right)=0, \\
\Gamma^{[2]}\left(v_{t}-f^{3} u_{x x}-f^{4} v_{x x}-u_{x}\left[f_{u}^{3} u_{x}+f_{v}^{3} v_{x}\right]-v_{x}\left[f_{u}^{4} u_{x}+f_{v}^{4} v_{x}\right]\right)=0
\end{gathered}
$$

whenever system (1) is satisfied and where

$$
\Gamma^{[2]}=\Gamma+\zeta_{1}^{1} \partial_{u_{t}}+\zeta_{2}^{1} \partial_{u_{x}}+\zeta_{1}^{2} \partial_{v_{t}}+\zeta_{2}^{2} \partial_{v_{x}}+\zeta_{22}^{1} \partial_{u_{x x}}+\zeta_{22}^{2} \partial_{v_{x x}}
$$

The variables $\zeta_{j}^{i}$ are given by the prolongation formulae

$$
\begin{aligned}
\zeta_{1}^{1} & =D_{t}\left(\eta^{1}\right)-u_{t} D_{t}\left(\xi^{1}\right)-u_{x} D_{t}\left(\xi^{2}\right), \\
\zeta_{2}^{1} & =D_{x}\left(\eta^{1}\right)-u_{t} D_{x}\left(\xi^{1}\right)-u_{x} D_{x}\left(\xi^{2}\right), \\
\zeta_{1}^{2} & =D_{t}\left(\eta^{2}\right)-v_{t} D_{t}\left(\xi^{1}\right)-v_{x} D_{t}\left(\xi^{2}\right), \\
\zeta_{2}^{2} & =D_{x}\left(\eta^{2}\right)-v_{t} D_{x}\left(\xi^{1}\right)-v_{x} D_{x}\left(\xi^{2}\right), \\
\zeta_{22}^{1} & =D_{x}\left(\zeta_{2}^{1}\right)-u_{t x} D_{x}\left(\xi^{1}\right)-u_{x x} D_{x}\left(\xi^{2}\right), \\
\zeta_{22}^{2} & =D_{x}\left(\zeta_{2}^{2}\right)-v_{t x} D_{x}\left(\xi^{1}\right)-v_{x x} D_{x}\left(\xi^{2}\right),
\end{aligned}
$$

where $D_{t}$ and $D_{x}$ are the total derivative operators defined as follows

$$
D_{t}=\partial_{t}+u_{t} \partial_{u}+v_{t} \partial_{v}+\cdots, \quad D_{x}=\partial_{x}+u_{x} \partial_{u}+v_{x} \partial_{v}+\cdots
$$

With the help of program YaLie the generator of symmetry group for system (1) is of the form

$$
\Gamma=a(t) \partial_{t}+b(t, x) \partial_{x}+c(t, x, u, v) \partial_{u}+d(t, x, u, v) \partial_{v}
$$

where $a, \ldots, d$ are the smooth differentiable functions satisfying determining equations resulting from the invariance conditions (5).

It is not easy to employ the group classification procedure discussed in [17] to solve completely these determining equations. Therefore we opt for the approach mentioned earlier in the previous section to perform the complete group classification up to equivalence group (3). 


\section{CLASSIFICATION WITH RESPECT TO LOW-DIMENSIONAL LIE ALGEBRAS}

\subsection{One-dimensional Lie algebras}

A symmetry generator (9) can be transformed by the change of variables (3) into the form

$$
\bar{\Gamma}=a(t) \dot{T}(t) \partial_{\bar{t}}+b X^{\prime}(x) \partial_{\bar{x}}+\left(c U_{u}+d U_{v}\right) \partial_{\bar{u}}+\left(c V_{u}+d V_{v}\right) \partial_{\bar{v}},
$$

where the overdot and prime denote differentiation with respect to respective arguments and subscripts represent partial differentiation.

We follow an argument similar to that used in [3]. We consider the cases $a(t) \neq 0$ and $a(t)=0$. If $a(t) \neq 0$, then the choice in (3) of a function $T(t)$ such that $a(t) \dot{T}(t)=1$ and functions $X, U, V$ of the fundamental solutions of the system of PDEs

$$
b X^{\prime}=0, \quad c U_{u}+d U_{v}=0, \quad c V_{u}+d V_{v}=0,
$$

gives rise to $\Gamma \rightarrow \bar{\Gamma}=\partial_{\bar{t}}$.

If $a(t)=0$, then the choice in (3) of a particular solution of the PDE $b X^{\prime}=1$ as a function of $X$ and fundamental solutions of the PDEs $c U_{u}+d U_{v}=0, c V_{u}+d V_{v}=0$ as functions of $U$ and $V$ respectively, we have $\Gamma \rightarrow \bar{\Gamma}=\partial_{\bar{x}}$. Therefore, when we drop the bars, the symmetry operator (9) reduces to one of the canonical operators

$$
\Gamma=\partial_{t}, \quad \Gamma=\partial_{x}, \quad \Gamma=\partial_{u}, \quad \Gamma=\partial_{v} .
$$

Consequently there are four inequivalent realizations of one-dimensional Lie algebras denoted: $A_{1}^{1}=\left\langle\partial_{t}\right\rangle, A_{1}^{2}=\left\langle\partial_{x}\right\rangle, A_{1}^{3}=\left\langle\partial_{u}\right\rangle$ and $A_{1}^{4}=\left\langle\partial_{v}\right\rangle$. The superscript distinguishes one realization from the other while the subscript denotes the dimension of the Lie algebra.

\subsection{Two-dimensional Lie algebras}

There are two inequivalent two-dimensional solvable Lie algebras: $A_{2,1}:\left[e_{1}, e_{2}\right]=$ $0, A_{2,2}:\left[e_{1}, e_{2}\right]=e_{2}$. These algebras contain the $1 \mathrm{D}$ Lie algebras $A_{1}^{1}=\left\langle\partial_{t}\right\rangle$, $A_{1}^{2}=\left\langle\partial_{x}\right\rangle, A_{1}^{3}=\left\langle\partial_{u}\right\rangle$ and $A_{1}^{4}=\left\langle\partial_{v}\right\rangle$. Hence in search for realizations of the 2D Lie algebras there are four cases to consider: $\left\langle\partial_{t}, e_{2}\right\rangle,\left\langle\partial_{x}, e_{2}\right\rangle,\left\langle\partial_{u}, e_{2}\right\rangle$ and $\left\langle\partial_{v}, e_{2}\right\rangle$ where $e_{2}$ is of the form (9).

Consider firstly the Lie algebra $A_{2,1}$.

(a) $\left\langle\partial_{t}, e_{2}\right\rangle$ : The Lie Bracket $\left[e_{1}, e_{2}\right]=0$ yields

$$
e_{2}=b(x) \partial_{x}+c(x, u, v) \partial_{u}+d(x, u, v) \partial_{v} .
$$


We use the equivalence transformations (3) to simplify $e_{2}$. Also we need the equivalence group of $A_{1}^{1}=\left\langle\partial_{t}\right\rangle$ : i.e.,

$$
\partial_{\bar{t}}=\partial_{t}(\bar{t}) \partial_{\bar{t}}+\partial_{t}(\bar{x}) \partial_{\bar{x}}+\partial_{t}(\bar{u}) \partial_{\bar{u}}+\partial_{t}(\bar{v}) \partial_{\bar{v}}=\dot{T} \partial_{\bar{t}}
$$

Thus the equivalence transformations for $A_{1}^{1}$ are given by

$$
\mathcal{E}\left(\partial_{t}\right)=\left\{\bar{t}=t+a_{1}, \bar{x}=X(x), \bar{u}=U(u, v), \bar{v}=V(u, v)\right\} .
$$

By the use of these transformations $e_{2}$ is transformed into

$$
\bar{e}_{2}=b X_{x} \partial_{\bar{x}}+\left(c U_{u}+d U_{v}\right) \partial_{\bar{u}}+\left(c V_{u}+d V_{v}\right) \partial_{\bar{v}}
$$

According to (12) we have the following realizations of $2 \mathrm{D}$ Lie algebras: $A_{2,1}^{1}=$ $\left\langle\partial_{t}, \partial_{x}\right\rangle, \quad A_{2,1}^{2}=\left\langle\partial_{t}, \partial_{u}\right\rangle, \quad A_{2,1}^{3}=\left\langle\partial_{t}, \partial_{v}\right\rangle$.

(b) Likewise for the case $\left\langle\partial_{x}, e_{2}\right\rangle$ we obtain the realizations: $A_{2,1}^{4}=\left\langle\partial_{x}, \partial_{u}\right\rangle, A_{2,1}^{5}=$ $\left\langle\partial_{x}, \partial_{v}\right\rangle$. The cases $\left\langle\partial_{u}, e_{2}\right\rangle$ and $\left\langle\partial_{v}, e_{2}\right\rangle$ lead to the realization $A_{2,1}^{6}=\left\langle\partial_{u}, \partial_{v}\right\rangle$. This realization yields a trivial case (i.e. all the arbitrary functions are constants) hence it is not utilized for further classification.

Therefore we have six inequivalent realizations for the $2 \mathrm{D}$ Lie algebra $A_{2,1}$. The realizations of Lie algebras $A_{2,1}^{2}, A_{2,1}^{4}$ and $A_{2,1}^{3}, A_{2,1}^{5}$ imply that the arbitrary functions $f^{1}, \ldots, f^{4}$ are respectively independent of $u$ and $v$.

If we proceed in the same manner, the $2 \mathrm{D}$ Lie algebra $A_{2,2}$ has the realizations: $A_{2,2}^{3}=\left\langle-x \partial_{x}-u \partial_{u}, \partial_{x}\right\rangle$ and $A_{2,2}^{4}=\left\langle-x \partial_{x}-v \partial_{v}, \partial_{x}\right\rangle$. Their corresponding functional forms for the arbitrary functions are given by

$$
\begin{aligned}
& A_{2,2}^{3}: f^{1}=u^{2} F(v), \quad f^{2}=u^{3} F(v), \quad f^{3}=u F(v), \quad f^{4}=u^{2} F(v) . \\
& A_{2,2}^{4}: f^{1}=v^{2} F(u), \quad f^{2}=v F(u), \quad f^{3}=v^{3} F(u), \quad f^{4}=v^{2} F(u) .
\end{aligned}
$$

In general the arbitrary functions of their respective arguments are different.

When we look at the above functional forms of the diffusion coefficients, the cases $A_{2,2}^{3}$ and $A_{2,2}^{4}$ can be regarded as one case for a choice of equivalence transformations of the form

$$
\bar{t}=t, \quad \bar{x}=x, \quad \bar{u}=v, \quad \bar{v}=u .
$$

\subsection{Three-dimensional solvable Lie algebras}

The solvable Lie algebras can be classified into decomposable and nondecomposable Lie algebras (see Appendix in [3]). There are two realizations for the 3D decomposable Lie algebra $A_{3,1}$ obtained from the realization of the $2 \mathrm{D}$ Lie algebra $A_{2,1}^{1}$. They are $A_{3,1}^{1}=\left\langle\partial_{t}, \partial_{x}, \partial_{u}\right\rangle$ and $\tilde{A}_{3,1}^{1}=\left\langle\partial_{t}, \partial_{x}, \partial_{v}\right\rangle$. These realizations satisfy the consistency conditions for system (1) provided the diffusion coefficients are functions of $v$ and $u$ respectively. There are also two realizations for the $3 \mathrm{D}$ Lie algebra 
$A_{3,2}$. This Lie algebra contains the $2 \mathrm{D}$ Lie algebras $A_{2,2}^{3}$ and $A_{2,2}^{4}$ from which we obtain $\tilde{A}_{3,2}^{3}=\left\langle-x \partial_{x}-u \partial_{u}, \partial_{x}, u \partial_{u}+v \partial_{v}\right\rangle$ and $\tilde{A}_{3,2}^{4}=\left\langle-x \partial_{x}-v \partial_{v}, \partial_{x}, u \partial_{u}+v \partial_{v}\right\rangle$ respectively. Therefore the respective forms of the diffusion coefficients are

$$
\begin{aligned}
& \tilde{A}_{3,2}^{3}: f^{1}=k_{1}\left(\frac{u}{v}\right)^{2}, \quad f^{2}=k_{2}\left(\frac{u}{v}\right)^{3}, \quad f^{3}=k_{3}\left(\frac{u}{v}\right), \quad f^{4}=k_{4}\left(\frac{u}{v}\right)^{2}, \\
& \tilde{A}_{3,2}^{4}: f^{1}=\bar{k}_{1}\left(\frac{v}{u}\right)^{2}, \quad f^{2}=\bar{k}_{2}\left(\frac{v}{u}\right), \quad f^{3}=\bar{k}_{3}\left(\frac{v}{u}\right)^{3}, \quad f^{4}=\bar{k}_{4}\left(\frac{v}{u}\right)^{2},
\end{aligned}
$$

where $k_{1}, \ldots, k_{4}$ and $\bar{k}_{1}, \ldots, \bar{k}_{4}$ are the nonzero arbitrary constants. Likewise, these cases can be taken as one case if we make use of the change of variables (13).

Secondly we look at the 3D nondecomposable solvable Lie algebras. The results are summarized in Table 1. The Lie algebras that do not feature in this table of results imply that there are no realizations for such Lie algebras which satisfy the consistency conditions for system (1).

Table 1: 3D nondecomposable solvable Lie algebras.

\begin{tabular}{cccccc}
\hline \hline Algebra & $f^{1}$ & $f^{2}$ & $f^{3}$ & $f^{4}$ & Realization \\
\hline$A_{3,5}^{1}$ & $u F(v)$ & $u^{2} F(v)$ & $F(v)$ & $u F(v)$ & $\left\langle\partial_{t}, \partial_{x}, t \partial_{t}+x \partial_{x}+u \partial_{u}\right\rangle$ \\
$\tilde{A}_{3,5}^{2}$ & $K_{1} v^{-1}$ & $K_{2} v^{-1}$ & $K_{3} v^{-1}$ & $K_{4} v^{-1}$ & $\left\langle\partial_{t}, \partial_{u}, t \partial_{t}+u \partial_{u}+v \partial_{v}\right\rangle$ \\
$\tilde{A}_{3,5}^{4}$ & $K_{1} v^{2}$ & $K_{2} v^{2}$ & $K_{3} v^{2}$ & $K_{4} v^{2}$ & $\left\langle\partial_{x}, \partial_{u}, x \partial_{x}+u \partial_{u}+v \partial_{v}\right\rangle$ \\
$A_{3,6}^{1}$ & $u^{-3} F(v)$ & $u^{-2} F(v)$ & $u^{-4} F(v)$ & $u^{-3} F(v)$ & $\left\langle\partial_{t}, \partial_{x}, t \partial_{t}-x \partial_{x}+u \partial_{u}\right\rangle$ \\
$\tilde{A}_{3,6}^{2}$ & $K_{1} v^{-1}$ & $K_{2} v^{-3}$ & $K_{3} v$ & $K_{4} v^{-1}$ & $\left\langle\partial_{t}, \partial_{u}, t \partial_{t}-u \partial_{u}+v \partial_{v}\right\rangle$ \\
$\tilde{A}_{3,6}^{4}$ & $K_{1} v^{2}$ & $K_{2}$ & $K_{3} v^{4}$ & $K_{4} v^{2}$ & $\left\langle\partial_{x}, \partial_{u}, x \partial_{x}-u \partial_{u}+v \partial_{v}\right\rangle$ \\
$A_{3,7}^{1}$ & $u^{2 q-1} F(v)$ & $u^{2 q} F(v)$ & $u^{2(q-1)} F(v)$ & $u^{2 q-1} F(v)$ & $\left\langle\partial_{t}, \partial_{x}, t \partial_{t}+q x \partial_{x}+u \partial_{u}\right\rangle$ \\
$\hat{A}_{3,7}^{1}$ & $u^{2-q} F(v)$ & $u^{3-q} F(v)$ & $u^{1-q} F(v)$ & $u^{2-q} F(v)$ & $\left\langle\partial_{x}, \partial_{t}, q t \partial_{t}+x \partial_{x}+u \partial_{u}\right\rangle$ \\
$\tilde{A}_{3,7}^{2}$ & $K_{1} v^{-1}$ & $K_{2} v^{q-2}$ & $K_{3} v^{-q}$ & $K_{4} v^{-1}$ & $\left\langle\partial_{t}, \partial_{u}, t \partial_{t}+q u \partial_{u}+v \partial_{v}\right\rangle$ \\
$\bar{A}_{3,7}^{2}$ & $K_{1} v^{-q}$ & $K_{2} v^{-q}$ & $K_{3} v^{-q}$ & $K_{4} v^{-q}$ & $\left\langle\partial_{u}, \partial_{t}, q t \partial_{t}+u \partial_{u}+v \partial_{v}\right\rangle$ \\
$\tilde{A}_{3,7}^{4}$ & $K_{1} v^{2}$ & $K_{2} v^{q+1}$ & $K_{3} v^{3-q}$ & $K_{4} v^{2}$ & $\left\langle\partial_{x}, \partial_{u}, x \partial_{x}+q u \partial_{u}+v \partial_{v}\right\rangle$ \\
$\bar{A}_{3,7}^{4}$ & $K_{1} v^{2 q}$ & $K_{2} v^{2 q}$ & $K_{3} v^{2 q}$ & $K_{4} v^{2 q}$ & $\left\langle\partial_{u}, \partial_{x}, q x \partial_{x}+u \partial_{u}+v \partial_{v}\right\rangle$ \\
\hline \hline
\end{tabular}

The arbitrary functions $F(v)$ are in general different and $K_{i}$ are (generally different) arbitrary constants.

It should be noted that making use of the equivalence transformations (13), the arbitrary functions depend upon $u$ and the cases for which the diffusion coefficients are completely specified are in terms of $u$. This note applies to all the summarized results in the sequel. 


\section{COMPLETE GROUP CLASSIFICATION}

We proceed to the classification with respect to four-dimensional solvable Lie algebras, that is, we extend the realizations of the 3D Lie algebras obtained in the previous section. After this classification the functional forms of the arbitrary functions are in terms of the arbitrary constants only (c.f. Table 1). However, there is a need to investigate whether or not the realizations of both the $3 \mathrm{D}$ and $4 \mathrm{D}$ Lie algebras give the maximal symmetry Lie algebra.

Firstly we consider the decomposable 4D solvable Lie algebras. The extension of the realizations is possible for the $4 \mathrm{D}$ Lie algebra $4 A_{1}$ in the following cases:

$$
\begin{aligned}
& 4 A_{1}^{1}=\left\langle\partial_{t}, \partial_{x}, \partial_{u}, v \partial_{v}\right\rangle: f^{1}=\ell_{1}, \quad f^{2}=\ell_{2} v^{-1}, \quad f^{3}=\ell_{3} v, \quad f^{4}=\ell_{4}, \\
& 4 A_{1}^{2}=\left\langle\partial_{t}, \partial_{x}, u \partial_{u}, \partial_{v}\right\rangle: f^{1}=\bar{\ell}_{1}, \quad f^{2}=\bar{\ell}_{2} u, \quad f^{3}=\bar{\ell}_{3} u^{-1}, \quad f^{4}=\bar{\ell}_{4},
\end{aligned}
$$

for the nonzero arbitrary constants, $\ell_{1}, \ldots, \ell_{4}$ and $\bar{\ell}_{1}, \ldots, \bar{\ell}_{4}$, are arbitrary.

Now in Table 2 we present the realizations of $4 \mathrm{D}$ nondecomposable solvable Lie algebras which contain the 3D realizations and their corresponding forms of the diffusion coefficients.

Table 2: 4D non decomposable solvable Lie algebras.

\begin{tabular}{cccccc}
\hline \hline Algebra & $f^{1}$ & $f^{2}$ & $f^{3}$ & $f^{4}$ & Realization \\
\hline$A_{4,5}^{1}$ & $\lambda_{1} v^{2 q-1}$ & $\lambda_{2} v^{p+2 q-2}$ & $\lambda_{3} v^{2 q-p}$ & $\lambda_{4} v^{2 q-1}$ & $\left\langle\partial_{t}, \partial_{x}, \partial_{u}, t \partial_{t}+q x \partial_{x}+p u \partial_{u}+v \partial_{v}\right\rangle$ \\
$\tilde{A}_{4,5}^{1}$ & $\lambda_{1} v^{2 p-1}$ & $\lambda_{2} v^{2 p+q-2}$ & $\lambda_{3} v^{2 p-q}$ & $\lambda_{4} v^{2 p-1}$ & $\left\langle\partial_{t}, \partial_{u}, \partial_{x}, t \partial_{t}+p x \partial_{x}+q u \partial_{u}+v \partial_{v}\right\rangle$ \\
$\hat{A}_{4,5}^{1}$ & $\lambda_{1} v^{2-q}$ & $\lambda_{2} v^{p-q+1}$ & $\lambda_{3} v^{3-p-q}$ & $\lambda_{4} v^{2-q}$ & $\left\langle\partial_{x}, \partial_{t}, \partial_{u}, q t \partial_{t}+x \partial_{x}+p u \partial_{u}+v \partial_{v}\right\rangle$ \\
$\bar{A}_{4,5}^{1}$ & $\lambda_{1} v^{2-p}$ & $\lambda_{2} v^{1-p+q}$ & $\lambda_{3} v^{3-p-q}$ & $\lambda_{4} v^{2-p}$ & $\left\langle\partial_{x}, \partial_{u}, \partial_{t}, p t \partial_{t}+x \partial_{x}+q u \partial_{u}+v \partial_{v}\right\rangle$ \\
$\check{A}_{4,5}^{1}$ & $\lambda_{1} v^{2 p-q}$ & $\lambda_{2} v^{2 p-q}$ & $\lambda_{3} v^{2 p-q}$ & $\lambda_{4} v^{2 p-q}$ & $\left\langle\partial_{u}, \partial_{t}, \partial_{x}, q t \partial_{t}+p x \partial_{x}+u \partial_{u}+v \partial_{v}\right\rangle$ \\
$\dot{A}_{4,5}^{1}$ & $\lambda_{1} v^{2 q-p}$ & $\lambda_{2} v^{2 q-p}$ & $\lambda_{3} v^{2 q-p}$ & $\lambda_{4} v^{2 q-p}$ & $\left\langle\partial_{u}, \partial_{x}, \partial_{t}, p t \partial_{t}+q x \partial_{x}+u \partial_{u}+v \partial_{v}\right\rangle$ \\
\hline \hline
\end{tabular}

The $\lambda_{i}$ are arbitrary constants which are in general different.

\section{FURTHER ANALYSIS}

In order to investigate the maximal symmetry Lie algebra, we perform symmetry analysis of all the cases in Tables 1 and 2 for which the arbitrary functions are completely specified. The cases arising from the decomposable solvable Lie algebras are also taken into account.

We show the detailed calculations of one case for both the classification with respect to $3 \mathrm{D}$ and $4 \mathrm{D}$ Lie algebras and present the results only for the rest. 
Firstly we consider the $3 \mathrm{D}$ realization $\tilde{A}_{3,7}^{2}=\left\langle\partial_{t}, \partial_{u}, t \partial_{t}+q u \partial_{u}+v \partial_{v}\right\rangle$ from Table 1 . We proceed by substituting the corresponding forms of the arbitrary functions into the system (1) under consideration. Lie's algorithm yields the symmetry generator (9) the coordinates of which are of the form $a=a(t), b=b(t, x)$, $c=e(t, x) u+f(t, x), d=d(t, x, v)$ for arbitrary functions $e(t, x)$ and $f(t, x)$. The smooth differentiable functions, $a, b, d, e$ and $f$, satisfy the determining equations

$$
\begin{aligned}
K_{1} v\left(u e_{x}+f_{x}\right)+v^{q} K_{2}\left[v b_{x x}-2\left((q-2) d_{x}+v d_{x v}\right)\right] & =0, \\
K_{1}\left(u e_{x x}+f_{x x}\right)+K_{2} v^{q-1} d_{x x}-v\left(u e_{t}+f_{t}\right) & =0, \\
v\left(\dot{a}-2 b_{x}+d_{v}\right)-2 d & =0, \\
d-v \dot{a}+2 v b_{x} & =0, \\
(q-2) d+v\left(-e+\dot{a}-2 b_{x}+d_{v}\right) & =0, \\
v\left[(q-2) \dot{a}-(q-2) e+2(2-q) b_{x}+2(q-2) d_{v}+v d_{v v}\right] & \\
+\left(6-5 q+q^{2}\right) d & =0, \\
K_{1}\left(v b_{x x}+d_{x}-2 v e_{x}\right)-v^{2} b_{t} & =0, \\
v b_{x x}+q d_{x}-2 v e_{x} & =0, \\
v^{-(q+1)}\left[K_{3} v\left(u e_{x x}+f_{x x}\right)+K_{4} v^{q} d_{x x}\right]-d_{t} & =0, \\
v\left(\dot{a}-2 b_{x}+d_{v}-v d_{v v}\right)-2 d & =0, \\
(1+q) d-v\left(e+\dot{a}-2 b_{x}\right) & =0, \\
v\left(e+\dot{a}-2 b_{x}-d_{v}\right)-q d & =0, \\
K_{4}\left(v b_{x x}+2 d_{x}-2 v d_{x v}\right)-v^{2} b_{t} & =0 .
\end{aligned}
$$

In the above equations and throughout the overdot represents total derivative with respect to $t$ and the subscripts denote partial differentiation. The solution of Eqs. (14)-(26) is given by

$$
a=C_{3} t+C_{4}, b=C_{1} x+C_{2}, c=q\left(C_{3}-2 C_{1}\right) u+C_{5}, d=\left(C_{3}-2 C_{1}\right) v,
$$

where $C_{1}, \ldots, C_{5}$ are the arbitrary constants of integration.

Thus the symmetry Lie algebra for this case is spanned by the operators

$$
\Gamma_{1}=\partial_{t}, \Gamma_{2}=\partial_{x}, \Gamma_{3}=\partial_{u}, \Gamma_{4}=t \partial_{t}+q u \partial_{u}+v \partial_{v}, \Gamma_{5}=x \partial_{x}-2 q u \partial_{u}-2 v \partial_{v}
$$

The full symmetry Lie algebra for the rest of the cases that arise from symmetry analysis are presented below.

$\tilde{A}_{3,5}^{2}: \Gamma_{1}=\partial_{t}, \Gamma_{2}=\partial_{x}, \Gamma_{3}=\partial_{u}, \Gamma_{4}=t \partial_{t}+u \partial_{u}+v \partial_{v}, \Gamma_{5}=x \partial_{x}-2 u \partial_{u}-2 v \partial_{v}$.

$\tilde{A}_{3,5}^{4}: \Gamma_{1}=\partial_{t}, \Gamma_{2}=\partial_{x}, \Gamma_{3}=\partial_{u}, \Gamma_{4}=2 t \partial_{t}-u \partial_{u}-v \partial_{v}, \Gamma_{5}=x \partial_{x}+u \partial_{u}+v \partial_{v}$. 
$\tilde{A}_{3,6}^{2}: \Gamma_{1}=\partial_{t}, \Gamma_{2}=\partial_{x}, \Gamma_{3}=\partial_{u}, \Gamma_{4}=t \partial_{t}-u \partial_{u}+v \partial_{v}, \Gamma_{5}=x \partial_{x}+2 u \partial_{u}-2 v \partial_{v}$.
$\tilde{A}_{3,6}^{4}: \Gamma_{1}=\partial_{t}, \Gamma_{2}=\partial_{x}, \Gamma_{3}=\partial_{u}, \Gamma_{4}=2 t \partial_{t}+u \partial_{u}-v \partial_{v}, \Gamma_{5}=x \partial_{x}-u \partial_{u}+v \partial_{v}$.
$\tilde{A}_{3,7}^{2}: \Gamma_{1}=\partial_{t}, \Gamma_{2}=\partial_{x}, \Gamma_{3}=\partial_{u}, \Gamma_{4}=t \partial_{t}+q u \partial_{u}+v \partial_{v}, \Gamma_{5}=x \partial_{x}-2 q u \partial_{u}-2 v \partial_{v}$.
$\bar{A}_{3,7}^{2}: \Gamma_{1}=\partial_{t}, \Gamma_{2}=\partial_{x}, \Gamma_{3}=\partial_{u}, \Gamma_{4}=q t \partial_{t}+u \partial_{u}+v \partial_{v}, \Gamma_{5}=q x \partial_{x}-2 u \partial_{u}-2 v \partial_{v}$.
$\tilde{A}_{3,7}^{4}: \Gamma_{1}=\partial_{t}, \Gamma_{2}=\partial_{x}, \Gamma_{3}=\partial_{u}, \Gamma_{4}=2 t \partial_{t}-q u \partial_{u}-v \partial_{v}, \Gamma_{5}=x \partial_{x}+q u \partial_{u}+v \partial_{v}$.
$\bar{A}_{3,7}^{4}: \Gamma_{1}=\partial_{t}, \Gamma_{2}=\partial_{x}, \Gamma_{3}=\partial_{u}, \Gamma_{4}=2 q t \partial_{t}-u \partial_{u}-v \partial_{v}, \Gamma_{5}=q x \partial_{x}+u \partial_{u}+v \partial_{v}$.

For every case above the symmetry Lie algebra is spanned by the three operators from the $3 \mathrm{D}$ realization and two additional operators that arise from symmetry analysis.

Next we show the details of symmetry analysis for the case of $4 \mathrm{D}$ decomposable Lie algebra $4 A_{1}$ with the realization: $4 A_{1}^{1}=\left\langle\partial_{t}, \partial_{x}, \partial_{u}, v \partial_{v}\right\rangle$.

We proceed as in the previous symmetry analysis. Following the usual Lie's algorithm the coefficients of the symmetry generator (9) are of the form

$$
a=a(t), \quad b=\frac{1}{2} \dot{a} x+\bar{e}(t), \quad c=\bar{f}(t, x) u+\bar{g}(t, x), \quad d=d(t, x, v) .
$$

The arbitrary smooth functions $a, d, \bar{e}, \bar{f}$ and $\bar{g}$ from (28) satisfy the determining equations

$$
\begin{aligned}
v d_{x v}-d_{x} & =0, \\
\ell_{1} v\left(u \bar{f}_{x x}+\bar{g}_{x x}\right)+\ell_{2} d_{x x}-v\left(u \bar{f}_{t}+\bar{g}_{t}\right) & =0, \\
2 d+v\left(\bar{f}-2 d_{v}+v d_{v v}\right) & =0, \\
d+v\left(\bar{f}-d_{v}\right) & =0, \\
2 \bar{e}_{t}+x \ddot{a}+4 \ell_{1} \bar{f}_{x} & =0, \\
\ell_{4} d_{v v} & =0, \\
2 v \bar{f}_{x}+d_{x} & =0, \\
\ell_{3} v\left(u \bar{f}_{x x}+\bar{g}_{x x}\right)+\ell_{4} d_{x x}-d_{t} & =0, \\
\ell_{3} \bar{f} & =0, \\
2 \bar{e}_{t}+x \ddot{a}+2 \ell_{3}\left(u \bar{f}_{x}+\bar{g}_{x}\right)+4 \ell_{4} d_{x v} & =0 .
\end{aligned}
$$

Eventually the solution of the determining equations (29)-(38) is given by

$$
a=2 \bar{C}_{1} t+\bar{C}_{2}, \quad b=\bar{C}_{1} x+\bar{C}_{3}, \quad c=\bar{C}_{4}, \quad d=\bar{C}_{5} v,
$$

where $\bar{C}_{1}, \ldots, \bar{C}_{5}$ are arbitrary constants of integration.

Therefore for this case the symmetry Lie algebra is spanned by the operators $\Gamma_{1}=\partial_{t}, \Gamma_{2}=\partial_{x}, \Gamma_{3}=\partial_{u}, \Gamma_{4}=v \partial_{v}, \Gamma_{5}=2 t \partial_{t}+x \partial_{x}$. 
The symmetry Lie algebra for the other case of the 4D decomposable Lie algebra $4 A_{1}^{2}$ is spanned by

$$
\Gamma_{1}=\partial_{t}, \Gamma_{2}=\partial_{x}, \Gamma_{3}=u \partial_{u}, \Gamma_{4}=\partial_{v}, \Gamma_{5}=2 t \partial_{t}+x \partial_{x} .
$$

The maximal symmetry Lie algebras for every case in Table 2 of the four-dimensional nondecomposable Lie algebras are summarized as follows:

$$
\begin{aligned}
& A_{4,5}^{1} \cup\left\langle(2 q-1) t \partial_{t}-p u \partial_{u}-v \partial_{v},(2 q-1) x \partial_{x}+2 p u \partial_{u}+2 v \partial_{v}\right\rangle . \\
& \tilde{A}_{4,5}^{1} \cup\left\langle(2 p-1) t \partial_{t}-q u \partial_{u}-v \partial_{v},(2 p-1) x \partial_{x}+2 q u \partial_{u}+2 v \partial_{v}\right\rangle . \\
& \hat{A}_{4,5}^{1} \cup\left\langle(q-2) t \partial_{t}+p u \partial_{u}+v \partial_{v},(q-2) x \partial_{x}-2 p u \partial_{u}-2 v \partial_{v}\right\rangle . \\
& \bar{A}_{4,5}^{1} \cup\left\langle(p-2) t \partial_{t}+q u \partial_{u}+v \partial_{v},(p-2) x \partial_{x}-2 q u \partial_{u}-2 v \partial_{v}\right\rangle . \\
& \check{A}_{4,5}^{1} \cup\left\langle(2 p-q) t \partial_{t}-u \partial_{u}-v \partial_{v},(2 p-q) x \partial_{x}+2 u \partial_{u}+2 v \partial_{v}\right\rangle . \\
& \grave{A}_{4,5}^{1} \cup\left\langle(p-2 q) t \partial_{t}+u \partial_{u}+v \partial_{v},(p-2 q) x \partial_{x}-2 u \partial_{u}-2 v \partial_{v}\right\rangle .
\end{aligned}
$$

In all the above cases the symmetry Lie algebra is six-dimensional, four operators from the classification with respect to $4 \mathrm{D}$ Lie algebras and the additional two operators obtained via symmetry analysis. Moreover it can be seen that, if $p=q$, then a pair of similar cases is reduced to one case.

\section{CONCLUDING REMARKS}

We have managed to specify completely the functional forms of the diffusion coefficients through the classification with respect to the solvable Lie algebras of dimension up to four. In order to determine the maximal symmetry Lie algebra, we performed the symmetry analysis of individual cases for the realizations of the 3D and 4D Lie algebras in which the functional forms of the unknown functions are in terms of the arbitrary constants. In short the full classification has been achieved. The comparison with the previous studies of the model considered in $[9,11]$ can hardly be established. It should be noted that group classification using the other types of Lie algebras has not been considered in this work. These are semisimple Lie algebras and the algebras which are semidirect sum of semisimple algebras and solvable Lie algebras (c.f. Appendix [3]). The next step will be to find both the analytical and numerical solutions of the submodels.

Acknowledgements- M M acknowledges financial support from the National University of Lesotho, National Manpower Development Secretariat and University of the Witwatersrand.

\section{REFERENCES}

[1] M. Molati and F. M. Mahomed, A group classification of general second-order diffusion system, (Accepted: J. Phys. A: Math. Theor.). 
[2] R. Zhdanov and V. Lahno, Group classification of the heat conductivity equations with a nonlinear source, J. Phys. A: Math. Gen. 26, 7061-7076, 1999.

[3] P. Basarab-Horwath, V. Lahno and R. Zhdanov, The structure of Lie algebras and the classification problem for partial differential equations, Acta Applicandae Mathematicae 69, 43-94, 2001.

[4] F. M. Mahomed and P. G. L. Leach, Lie algebras associated with second-order ordinary differential equations, J. Math. Phys. 30, 2770-2777, 1989.

[5] G. M. Mubarakzyanov, On solvable Lie algebras (in Russian), Izv. Vys. Ucheb. Zaved. 32, 114-123, 1963.

[6] J. R. Philip and D. A. De Vries, Moisture movement in porous media under temperature gradients, Trans. Am. Geophys. Union 38, 222-232, 1957.

[7] D. A. De Vries, Simultaneous transfer of heat and moisture in porous media, Trans. Am. Geophys. Union 39, 909-916, 1958.

[8] W. A. Jury, J. Letey and L. H. Stolzy, Flow of water and energy under desert conditions, in: D. D. Evans, J. L. Thames (Eds.), Water in Desert Ecosystems, Dowden, Hutchinson and Ross, Stroudsburg, PA, 92-113, 1981.

[9] V. A. Baikov, A. V. Gladkov and R. J. Wiltshire, Lie symmetry classification analysis for nonlinear coupled diffusion, J. Phys. A: Math. Gen. 31, 7483-7499, 1998.

[10] C. Sophocleous and R. J. Wiltshire, On linearizing systems diffusion equations, SIGMA, 2, paper 004, 2006.

[11] R. J. Wilshire, The use of Lie transformation groups in the solution of the coupled diffusion equation, J. Phys. A: Math. Gen. 27, 7821-7829, 1994.

[12] L. V. Ovsjannikov, Group Properties of Differential Equations (in Russian), English translation by G. W. Bluman, Novosibirsk: USSR Acad. of Sci., Siberian Section, 1962.

[13] C. Sophocleous and R. J. Wiltshire, Linearisation and potential symmetries of certain systems of diffusion equations, Physica A 370, 329-345, 2006.

[14] J. M. Di'az, Short guide to Yalie, Yet another Lie Mathematica package for Lie symmetries, 
http://www.mathsource.com/MathSource/Applications/Mathematics/ 0211-778/YaLie.ps

[15] G. W. Bluman and S. Kumei, Symmetries and Differential Equations, SpringerVerlag, New York, 1989.

[16] P. J. Olver, Applications of Lie Groups to Differential Equations, Springer, New York, 1986.

[17] L. V. Ovsiannikov, Group Analysis of Differential Equations, Academic, New York, 1982. 